\title{
Some Characteristic of Factorial State Space
}

\author{
Dr. Mohammad abid ansari \\ Department of Mathematics, T.N.B. College, T. M. Bhagalpur University, Bhagalpur-812007 (Bihar)
}

\begin{abstract}
In this paper we present a characteristic of a factorial state space with $C^{*}$ - algebra and von Neumann algebra. We also discuss some theorems on $C^{*}$ - algebra and von - Neumann algebra.
\end{abstract}

\section{Introduction}

Factor: - A $w^{*}$ - algebra $M$ is called a factor if its centre consists of the scalar multiples of unity only. Central Projection: A projection $Z$ is a $w^{*}$ - algebra $M$ is called a central projection if $X Z=Z(X Z)=Z \times Z$ and $Z X=(Z X) Z=Z \times Z$ for all $x \in M$.

Central Support: Let $L$ be the centre of a $w^{*}-\operatorname{algebra} M$. then $L$ is $\sigma\left(M, M^{*}\right)$ - closed. Let $P$ be a projection in $M$. Then there exists a least central projection in $M$ containg $P$. We call this central projection the central support (or envelop) of $P$ and is denoted by $C(P)$.

A non-zero projection $P$ is a $w^{*}$ - algebra $M$ is said to be abelian if $P M P$ is commutative.

Varieties of $w^{*}$ - algebra : - A $w^{*}$ - algebra is said to be of type 1, if every non-zero central projection contain an abelian projection of type 11 , if it is semi finite and does not contain any abelian projection of type 11, if it is finit and does not contain any abelian projection of type 111, if it is purely infinite. A properly infinite type 11 $w^{*}$ - algebra is said to be of type $11_{\infty}$.

Central $C^{*}$ - algebra: - A $c^{*}$ - algebra $A$ with centre $L$ is said to be a central if it satisfies the following two condition.

(i) No Primitive ideal of $A$ contain $L$

(ii) If $P_{1}, P_{2} \in$ Prime (A) and $P_{1} \cap L=P_{2} \cap L$ then $P_{1}=P_{2}$

Glimm Ideal: - Let A be a von Neumann algebra with centre L, Let $\mathrm{m}$ be a maximal ideal of L and Let $|\mathrm{m}|$ be the smallest ideal of A containing $\mathrm{m}$. Then $|\mathrm{m}|$ is said to be Glim ideal of A. It is well known that $|\mathrm{m}|$ is always a primitive ideal of $\mathrm{A}$.

Factorial States: - A state $\phi$ on a $C^{*}$ - algebra $A$ is said to be factorial if the weak Closure $\overline{\pi_{\phi}(A)}$ of $\pi_{\phi}(A)$ is a factor where $(H \phi, \pi \phi, \phi)$ is the GNS representation associated with the state $\phi$.

The set of all factorial states of $A$ is denoted by $F_{A}$ and $\overline{F_{A}}$ is called the factorial state space of A. It is important to note that $\overline{P_{A}} \subseteq F_{A}$, i.e. every pure state is a factorial state.

The factorial states associated with $w^{*}$ - algebra of types is type 11 and type 111 are called factorial states of type 1 , type 11 and type 111 respectively.

Let $\mathrm{F}_{1, \mathrm{~A}}, \mathrm{~F}_{2, \mathrm{~A}}$ and $\mathrm{F}_{3, \mathrm{~A}}$ denotes the sets of factorial states of type 1, type 11 and type 111 respectively.

\section{Theorems On $C^{*}$ - Algebra And Von - Neumann Algebra}

Theorem (1.1): Let A be a $c^{*}$ - algebra. Then the following statements are equivalent.
(i) A is prime
(ii) $\overline{F_{1, A}} \supseteq S_{A}$
(iii) $\overline{F_{A}} \supseteq S_{A}$ 
Now we prove the following result.

Theorem (1.2) : Let $\phi$ be a states on a $C^{*}$ - algebra A such that ker $\pi_{\phi}$ contains a prime ideal of A. Then $\phi \in \overline{F_{A}}$.

Proof : - Suppose that $J$ is a prime ideal of $A$ is ker $\pi_{\phi}$. Then $\phi$ can be represented as $\phi=\mid 0 \phi$ where $\phi$ is the canonical $*$ - homomorphism of $\mathrm{A}$ on to $\mathrm{A} / \mathrm{J}$ and $\psi \in S(A / J)$. It follows that $\psi \in \overline{F_{A / J}}$. Hence $\phi \in \overline{F_{A}}$.

Factorial state space characterized with $C^{*}$ - algebra and van Neumann algebra. Then the following results are as follows.

Theorem (1.3) : Let A be a Von - Neumann algebra with centre $L$. Then for $f \in S_{A}$ the following conditions are equivalent.

(a) $f \in \overline{F_{A}}$

(b) $\operatorname{Ker} \pi_{f}$ contain a Glimm ideal of $A$.

(c) $\operatorname{Ker} \pi_{f}$ contain a prime ideal of $A$.

(d) $\operatorname{Ker} \pi_{f}$ contain a primitive ideal of $A$.

Theorem (1.4) : Let A be a central $\mathrm{C}^{*}$ - algebra and let $f \in S_{A}$.

Then the following condition are equivalent.

(a) $f \in \overline{F_{A}}$

(b) Ker $\pi_{f}$ contain a prime ideal.

(c) $\operatorname{Ker} \pi_{f}$ contain a primitive ideal.

And further if $\mathrm{A}$ is non-unital then $\overline{F_{A}}=\{0\} \cup\left\{\overline{F_{A}} \cap S_{A}\right\}$.

\section{Reference}

[1]. Archbold, R.J.: On factorial states of operator algebra; J. Fuc. Ana., 55 (1984) No. 1, PP. 25-38.

[2]. Baty, C.J.K. : Nuctear faces of state spaces of $c^{*}$ - algebra, Proc, Amer. Math. Soc., 86 (1982), No. 2, PP. 273-278.

[3]. Sze-kai, Tsui : Factor state extension on nuclear C* - algebra, yokohoma Math. J., 29 (1981) No. 2, PP. 157-160.

[4]. Archbold, R.J.: Extension of factorial states of $C^{*}$ - algebra, J. Funct. Ana., 63(1985) Pp. 86-100.

[5]. Alfsen, E.M., Hanche-Olsen, H., Shultz, F.W., State spaces of C*-algebras, Acta Math., 144 (1980) $267-305$.

[6]. Glimm, J.; Astone-weierstrass theorem for C*-algebras, Ann. of Math., 72 (1960) 216-224.

[7]. Glimm, J. Type $1 \mathrm{C}^{*}$-algebras, Ann. of Math., 73(1961) 572-612.

[8]. Kadison, R.V., States and representations, Trans. Amer. Math. Soc., 103 (1962), 304-319.

[9]. Kaplansky, I., The structure of certain operator algebras, Trans. Amer. Math. Soc., 70 (1951) 219-255

[10]. Longo, R., Solution of the factorial stone-weierstrass conjecture. An application of standard split $\mathrm{W}^{*}$-inclusions, Invent. Math., 76 (1984), 145-155.

[11]. Popa, S., Semi regular abelian *-sub algebras and the solution to the factor state stone-weierstrass problem, Invent. Math., 76(1984), 157-161.

[12]. Take Saki, M., Theory of operator algebras I, Spring-Verlag, Berlin Heidelberg New York, 1979.

[13]. Wright, S., On factorial states, Rocky Mounteni J. Math., 12 (1982) 569-579.

[14]. S. Sakai, $C^{*}$-algebras and $W^{*}$ - algebras, Springer, New York, 1971. 